\title{
Fecal microbiome of growing pigs fed a cereal based diet including chicory (Cichorium intybus L.) or ribwort (Plantago lanceolata L.) forage
}

Johan Dicksved ${ }^{1,2^{*}}$, Janet K. Jansson ${ }^{3}$ and Jan Erik Lindberg ${ }^{1}$

\begin{abstract}
Background: The purpose of this study was to investigate how inclusion of chicory forage or ribwort forage in a cereal-based diet influenced the fecal microbial community (microbiome) in newly weaned (35 days of age) piglets. The piglets were fed a cereal-based diet without (B) and with inclusion (80 and $160 \mathrm{~g} / \mathrm{kg}$ air-dry forage) of vegetative shoots of chicory $(C)$ and leaves of ribwort $(\mathrm{R})$ forage in a 35-day growth trial. Fecal samples were collected at the start (D0), 17 (D17) and 35 (D35) days after weaning and profiles of the microbial consortia were generated using terminal restriction fragment length polymorphism (T-RFLP). 454-FLX pyrosequencing of 165 rRNA gene amplicons was used to analyze the microbial composition in a subset of the samples already analyzed with T-RFLP.
\end{abstract}

Results: The microbial clustering pattern was primarily dependent on age of the pigs, but diet effects could also be observed. Lactobacilli and enterobacteria were more abundant at D0, whereas the genera Streptococcus, Treponema, Clostridium, Clostridiaceae1 and Coprococcus were present in higher abundances at D35. Pigs fed ribwort had an increased abundance of sequences classified as Treponema and a reduction in lactobacilli. However, the abundance of Prevotellaceae increased with age in on both the chicory and the ribwort diet. Moreover, there were significant correlations between the abundance of Bacteroides and the digested amount of galactose, uronic acids and total non-starch polysaccharides, and between the abundance of Bacteroidales and the digested amount of xylose.

Conclusion: This study demonstrated that both chicory and ribwort inclusion in the diet of newly weaned pigs influenced the composition of the fecal microbiota and that digestion of specific dietary components was correlated with species composition of the microbiota. Moreover, this study showed that the gut will be exposed to a dramatic shift in the microbial community structure several weeks after weaning.

Keywords: Amplicon sequencing, Chicory, Microbiome, Ribwort, Uronic acid, Weaning, $16 \mathrm{~S}$

\section{Background}

In order to maintain normal physiological functions in the digestive tract of pigs, a minimum level of fiber has to be included in the diet [1]. Moreover, by increasing the fiber level in the diet of weaned piglets, the $\mathrm{pH}$ in the hindgut is reduced [2] and the content of organic acids in the stomach and the ileum is increased $[2,3]$.

\footnotetext{
* Correspondence: johan.dicksved@slu.se

'Department of Animal Nutrition and Management, Swedish University of Agricultural Sciences, P.O. Box 7024, SE75007 Uppsala, Sweden

${ }^{2}$ Department of Microbiology, Swedish University of Agricultural Sciences,

P.O. Box 7025, SE75007 Uppsala, Sweden

Full list of author information is available at the end of the article
}

These changes in the gut environment, induced by fiber inclusion, indicates a shift in dominating bacterial population which may impair the conditions for pathogenic bacteria and may be more beneficial for maintaining gut health $[1,4]$. Fiber properties (soluble vs. insoluble) and age of the pig will modulate the impact of fiber level on the gut environment [2]. Soluble fiber is well digested by both growing pigs and sows, whereas sows have a higher capacity to digest insoluble fiber [5].

Chicory (Cichorium intybus L.) and ribwort (Plantago lanceolata L.) are dicotelydenous herbs with a high content of uronic acid (80-90 g per kg dry matter) of 
which approximately $80 \%$ is soluble. Uronic acid in dicotelydenous plants derives from galactosyluronic acid that is the building block in pectins [6]. Uronic acid has a high digestibility in forage crops fed to growing pigs [7, 8]. Moreover, pectin substances from sugar beet pulp have been shown to influence the gut microbial ecosystem, in particular by increasing the fecal Lactobacillus counts [9], and is therefore a very interesting fiber component in piglet nutrition.

We have previously shown that inclusion of chicory in the diet influenced the intestinal micro-environment and the microbiota in pigs [10-12]. For example, inclusion of chicory forage was associated with higher abundance of ileal lactobacilli and colonic butyrate producing bacteria [11]. In addition, chicory forage inclusion influenced the relative abundance of Prevotella, but the change in abundance was dependent on species of Prevotella [10, 12]. We also found correlations between specific bacterial groups and short chain fatty acid (SCFA) profiles, which shows that inclusion of chicory is influencing the intestinal micro-environment. However, less is known about ribwort inclusion and its influence on the microbiota. Ribwort forage contains a range of bioactive and antimicrobial compounds that may influence the microbiota [13].

The recent technological development of the Next Generation Sequencing platforms has facilitated a deeper analysis of the gut microbiota composition, more recently referred to as "microbiome". The aim of this experiment was therefore to characterize the post weaning gut microbiome and to get a deeper understanding of how inclusion of chicory and ribwort forage in a cereal-based diet influences the microbiome in weaned piglets. Furthermore, we aimed to identify correlations between dietary components and the composition of the intestinal microbiome.

\section{Methods}

\section{Experimental setup}

The study included 195 -wk old weaned and castrated male piglets (Swedish Landrace $\times$ Yorkshire) used in a growth trial. The pigs originated from five different litters and had a live weight of $11.7 \mathrm{~kg}$ (s.d. $0.8 \mathrm{~kg}$ ) at the start of the experiment. The piglets were purchased from a herd free from diseases according to the A-list of International Office of Epizootics [14] and were housed individually in pens equipped with a rubber mat, urine drainage and no bedding.

Piglets had ad libitum access to feed and water throughout the experiment, except for the first days when the feed allowance was restricted. The experimental diets comprised a cereal-based basal diet (B) and two diets composed to contain 80 and $160 \mathrm{~g} / \mathrm{kg}$ air-dry forage made from vegetative shoots of chicory $(\mathrm{C} 80, \mathrm{C} 160)$ and leaves of ribwort (R80, R160), respectively. The basal diet was composed of ground cereals (wheat, barley and oats), milled through a 5-mm screen, supplemented with protein, amino acids, mineral and vitamins to meet nutritional requirements of piglets (Table 1). In diets with chicory and ribwort inclusion, the cereal mixture was substituted with the herbs on an air-dry basis (Table 1). The herbs where harvested at the vegetative stage (September) with a stubble height of $c .5 \mathrm{~cm}$ and dried with forced air at $30{ }^{\circ} \mathrm{C}$ for a week and milled through a $5-\mathrm{mm}$ screen before mixing with the other feed ingredients.

The experiment was organized according to a randomized block design, with three replicates for the low inclusion level of forage, four replicates for the highest inclusion level of forage and five replicates for basal diet (Table 2). Fecal samples were collected at the start of the experiment (D 0), after $17 \mathrm{~d}$ (D 17) and after $35 \mathrm{~d}$ (D35).

The experiment was carried out at the Swedish University of Agricultural Sciences (SLU) and was approved by the ethical committee of the Uppsala region.

\section{Chemical composition and digestibility of diets}

Chemical composition of the experimental diets (Table 3 ) as well as the digestibility of the dietary components had been characterized previously [7]. The chemical analysis included quantification of total soluble and insoluble non-starch polysaccharides (NSP) and their constituent sugars including uronic acids. The digested amount of arabinose, xylose, mannose, galactose, glucose, uronic acids, and total NSP was based on the intake and digestibility of each component.

Table 1 Ingredient composition $(\mathrm{g} / \mathrm{kg})$ of the experimental diets

\begin{tabular}{|c|c|c|c|c|c|}
\hline \multirow{2}{*}{ Ingredients } & \multirow{2}{*}{ Basal } & \multicolumn{2}{|c|}{ Chicory forage } & \multicolumn{2}{|c|}{ Ribwort forage } \\
\hline & & 80 & 160 & 80 & 160 \\
\hline Wheat & 400 & 360 & 320 & 360 & 320 \\
\hline Barley & 300 & 270 & 240 & 270 & 240 \\
\hline Oat & 100 & 90 & 80 & 90 & 80 \\
\hline Chicory forage & - & 80 & 160 & - & - \\
\hline Ribwort forage & - & - & - & 80 & 160 \\
\hline Fish meal & 30 & 30 & 30 & 30 & 30 \\
\hline Potato protein & 50 & 50 & 50 & 50 & 50 \\
\hline Casein & 40 & 40 & 40 & 40 & 40 \\
\hline Sugar & 40 & 40 & 40 & 40 & 40 \\
\hline Vitamin-mineral premix ${ }^{1}$ & 11 & 11 & 11 & 11 & 11 \\
\hline Others $^{2}$ & 29 & 29 & 29 & 29 & 29 \\
\hline
\end{tabular}

${ }^{1}$ Content/kg premix: vitamins (mg): A 1,000,000 IE, D 100,000 IE, E 6,000, K3 200, B1 200, B2 400, B6 300, B12 2, panthothenic acid 1,500, niacin 2,000, biotin 25. Minerals (mg): Fe 4,000, Cu 1,000, Mn 2,000, Zn 7,000, I 30, Se 35. ${ }^{2}$ Content $/ \mathrm{kg}$ others: lysine 1.2 , methionine 0.08 , threonine 0.03 , dibasic-calcium phosphate 20, Ca-carbonate $2.5, \mathrm{NaCl} 2.5, \mathrm{FeSO}_{4}+4 \mathrm{H}_{2} \mathrm{O} 0.24$, titanium (IV) oxide 2.5 
Table 2 Performance of weaned piglets and indication of samples used for T-RFLP and 454-pyrosequencing analysis

\begin{tabular}{llllll}
\hline Items & Number & Weight gain, g/day & Feed intake, g/day & T-RFLP (D0:D17:D35) & 454-Seq. (D0:D17:D35) \\
\hline Control & 5 & $690^{\mathrm{a}}$ & $1135^{\mathrm{a}}$ & $5: 5: 5$ & $3: 0: 3$ \\
Chicory 80 & 3 & $657^{\mathrm{a}}$ & $1181^{\mathrm{a}}$ & $3: 3: 3$ & $0: 0: 0$ \\
Chicory 160 & 4 & $629^{\mathrm{a}}$ & $1143^{\mathrm{a}}$ & $4: 4: 4$ & $3: 0: 3$ \\
Ribwort 80 & 3 & $640^{\mathrm{a}}$ & $1101^{\mathrm{a}}$ & $3: 3: 3$ & $0: 0: 0$ \\
Ribwort 160 & 4 & $528^{\mathrm{b}}$ & $970^{\mathrm{b}}$ & $4: 4: 4$ & $3: 0: 3$ \\
\hline
\end{tabular}

Different letters within column indicates a significant difference at $P<0.05$

\section{Terminal-restriction fragment length polymorphism (T-RFLP) analyses}

DNA was isolated from fecal samples in triplicates according to the method described by Leser et al. 2002 [15]. The 16S rRNA genes were PCR amplified from each DNA extract using the general bacterial primers Bact-8 F (5' -AGAGTTTGATCCTGGCTCAG-3') [16], $5^{\prime}$ end-labeled with 6-carboxyfluorescein (6-FAM), and 926r (5'-CCGTCAATTCCTTTRAGTTT-3') [17] under conditions described elsewhere [18]. DNA product amounts and sizes were confirmed by agarose gel

Table 3 Chemical composition ( $\mathrm{g} / \mathrm{kg}$ dry matter) of basal diet, chicory forage and ribwort forage

\begin{tabular}{|c|c|c|c|}
\hline Items & Basal diet (B) & Chicory forage (C) & Ribwort forage (R) \\
\hline Ash & 67.0 & 255.6 & 133.6 \\
\hline Crude protein & 211.4 & 195.2 & 169.4 \\
\hline Crude fat & 26.9 & 15.6 & 11.1 \\
\hline Starch & 463.0 & 24.5 & 9.5 \\
\hline WSC & 60.5 & 11.5 & 42.0 \\
\hline NDF & 107.6 & 268.0 & 352.0 \\
\hline \multicolumn{4}{|l|}{ NSP } \\
\hline Total & 187.2 & 311.0 & 308.5 \\
\hline Insoluble & 142.6 & 194.9 & 229.5 \\
\hline \multicolumn{4}{|l|}{ Glucose } \\
\hline Total & 89.4 & 133.7 & 134.9 \\
\hline Insoluble & 56.9 & 118.3 & 134.9 \\
\hline \multicolumn{4}{|l|}{ Arabinose } \\
\hline Total & 25.0 & 13.4 & 17.8 \\
\hline Insoluble & 22.4 & 5.6 & 10.4 \\
\hline \multicolumn{4}{|l|}{ Xylose } \\
\hline Total & 56.7 & 31.1 & 32.1 \\
\hline Insoluble & 56.2 & 27.2 & 26.8 \\
\hline \multicolumn{4}{|l|}{ Uronic acid } \\
\hline Total & 7.4 & 97.0 & 88.3 \\
\hline Insoluble & 4.0 & 21.2 & 19.9 \\
\hline Klason Lignin & 34.9 & 107.2 & 77.7 \\
\hline Dietary fibre & 222.0 & 418.4 & 386.2 \\
\hline
\end{tabular}

Abbreviations: WSC water soluble carbohydrates (free glucose + free fructose + sucrose + fructan), NDF neutral detergent fibre, NSP non-starch polysaccharides electrophoresis using GeneRuler 100 bp DNA ladder Plus (Fermentas Life Sciences, Burlington, Canada) as a size marker.

PCR products were digested with restriction enzyme HaeIII and the resulting fragments were separated on an ABI 3700 capillary sequencer (Applied Biosystems, Foster City, CA). The sizes of the fluorescently labelled fragments were determined by comparison with the internal GS ROX-500 size standard (Applied Biosystems). The T-RFLP electropherograms were imaged using the Peak scanner software (Applied Biosystems) and relative peak areas of each terminal restriction fragment (TRF) were determined by dividing the area of the peak of interest by the total area of peaks, using 50 and $500 \mathrm{bp}$ lower and upper threshold values, respectively. Data was normalized by applying a threshold value for relative abundance at $0.5 \%$, and only TRFs with higher relative abundances were included in the remaining analyses.

\section{4-pyrosequencing analysis}

The pig fecal microbiome was characterized with higher resolution in a subset of the pigs by 454-pyrosequencing (Table 2). The (V5 and V6) variable regions of the $16 \mathrm{~S}$ rRNA gene were amplified by PCR using forward primer (784f 5' - AGGATTAGATACCCTGGTA 3') and reverse primer (1061r 5' CRRCACGAGCTGACGAC 3'). The reverse primer was tagged with 1 of 4 labels (CGAT, CATG, CTGA and CGTA) at the $5^{\prime}$ end along with the adaptor sequence (5' - GCCTCCCTCGCGCCATCAG 3') to allow 4 samples to be included in a single 454-FLX pyrosequencing lane as previously described [19]. Two microliters of DNA was added to each $25 \mu \mathrm{L}$ PCR reaction containing $2.5 \mu \mathrm{L} 10 \times$ PCR buffer (Amersham Biosciences, Piscataway, NJ), $1 \mu \mathrm{L}$ BSA $(10 \mathrm{mg} / \mathrm{mL})$ (Amersham Biosciences), $1 \mu \mathrm{L}$ dNTP (5 mmol/L), $0.25 \mu \mathrm{L}$ Taq Polymerase (5 U / $\mu \mathrm{L}$ ) (Amersham Biosciences) and $1 \mu \mathrm{L}$ of each primer $(10 \mu \mathrm{mol} / \mathrm{L})$ (Scandinavian Gene Synthesis, Köping, Sweden). PCR reactions were carried out on a GeneAmp (Applied Biosystems, Foster City, CA) PCR system (5 min at $94{ }^{\circ} \mathrm{C}, 30$ cycles of $94{ }^{\circ} \mathrm{C}$ for $45 \mathrm{~s}, 55{ }^{\circ} \mathrm{C}$ for $40 \mathrm{~s}$ and 72 for $1 \mathrm{~min}$, and a final extension of $72{ }^{\circ} \mathrm{C}$ for $7 \mathrm{~min}$ ). Triplicate PCRs were pooled and $60 \mu \mathrm{L}$ were run on $1 \%$ agarose gels at $80 \mathrm{~V}$ for $1.5 \mathrm{~h}$. PCR products of the appropriate 
size (Approx. 340 bp) were gel purified (QIAquick Gel Extraction Kit, Qiagen, Gmbh, Germany) and eluted in $50 \mu \mathrm{L}$ of elution buffer. DNA quality was assessed on a Bioanalyzer 2100 (Agilent Technologies, Santa Clara, CA). DNA concentration was measured on a NanoDrop ND-1000 (NanoDrop Technologies, Wilmington, DE) and $25 \mathrm{ng}$ of four samples, labeled with different tag sequences, were pooled and diluted in water for a total of $100 \mathrm{ng}$ in $10 \mu \mathrm{L}$. Pyrosequencing was performed on a 454 Life Sciences Genome Sequencer FLX machine (Roche), at the Swedish Institute for Infectious Disease Control, Solna, Sweden.

\section{Taxonomic analysis}

Sequences were checked for quality and sequences that were less than $200 \mathrm{bp}$ in length, that contained incorrect primer sequences, or that contained more than $1 \mathrm{am}$ biguous base were discarded. Assignment of sequences to samples was based on the 4-bp barcode. Remaining sequences were then subjected to complete linkage clustering using the pyrosequencing pipeline at RDP-X using a conservative $5 \%$ dissimilarity to define operational taxonomic units (OTUs) because of the short sequence length. The most abundant sequence from each OTU was selected as a representative sequence and was taxonomically classified by BLAST searching against a local BLAST database comprised of 269,420 bacterial $16 \mathrm{~S}$ rRNA gene sequences longer than 1,200 bp with good Pintail scores from RDP v. 10.7. The OTU inherited the taxonomy (down to genus level) of the best scoring RDP hit fulfilling the criteria of $\geq 95 \%$ identity over an alignment of length $\geq 180 \mathrm{bp}$.

\section{Statistical analysis}

To visualize time or diet related effects in composition of the microbiota, relative abundance values and sizes of T-RFLP fragments were analyzed with principal component analysis (PCA) using the software Canoco (version 4.5, Microcomputer Power Ithaca, NY, USA). For the 454 data, principal coordinate analysis (PCoA) based on Bray Curtis distances were used to monitor clustering pattern of the microbial architecture using the software PAST [20]. To identify specific taxa that correlated with diet or time, statistical analyses were performed using GLM in SAS (SAS Institute, Cary, NC, USA, version 9.1). Pearson correlation analysis was used to identify correlations between digested amount of dietary components and the abundance of microbial taxa. The level of significance was set at $P<0.05$ and the Benjamini and Hochberg method was used to account for multiple comparisons, based on global $P$ values of the variables compared [21].

\section{Results}

\section{Pig performance}

Herb inclusion affected $(P<0.05)$ the average daily feed intake during the experiment (day $0-35$; Table 2), with lower intake for the diet (R160) with the highest ribwort inclusion than for the other diets [7]. Inclusion of chicory did however not impair feed intake compared with the basal diet. Moreover, as a consequence of the lower feed intake on the diet with the highest inclusion of ribwort, the daily weight gain was lower $(P<0.05)$ than for the other diets [7]. There was no negative impact on the daily weight gain of including chicory in the diet.

\section{T-RFLP analysis of the fecal microbiome}

Profiles of the microbial consortia in fecal samples were generated using T-RFLP. Principal component analysis was used to identify patterns in the microbiome that could be explained by factors such as time or diet effects. The PCA showed a clustering pattern that largely was dependent on age of the pigs (Fig. 1). All samples collected at weaning (D 0) clustered separately from the samples collected at D 17 and D 35. There was a large variation in microbial composition between individual pigs at weaning, and the samples were subsequently spread out along the first principal component (PC 1). No apparent clustering pattern of diet effects could be visualized in the PCA scatter plots (Fig. 1).

\section{Barcoded 454 pyrosequencing analysis of the fecal microbiome}

Pyrosequencing of $16 \mathrm{~S}$ rRNA gene amplicons was used to analyze the microbial composition in a subset of the samples that had already been analyzed with T-RFLP for a more detailed view of the microbial composition. $16 \mathrm{~S}$ data was obtained from nine pigs from samples collected both at D 0 and D 35 (Table 2). After quality filtering, 31,620 sequences were obtained, with an average of 1,757 sequences per sample (range 1,386-2,095). Analysis of the sequence data revealed a large individual variation between pigs but also that the fecal microbiome at weaning and 35 days after weaning was dominated by the same main phyla, primarily members of the Firmicutes (F) and Bacteroidetes (B) phyla. These were mainly dominated by the Lachnospiraceae (F), Ruminococcaceae (F), Lactobacillaceae (F), Streptococcaceae (F) and Prevotellaceae (B) families. In addition, a large fraction of the sequences could not be matched to the sequences in the public databases indicating presence of undescribed species.

\section{Development of the post weaning microbiome}

Principal coordinate analysis (PCoA) based on Bray Curtis distance metrics was used to visualize clustering patterns in the $16 \mathrm{~S}$ data. The samples arranged into two clusters and in agreement with the T-RFLP data analysis 


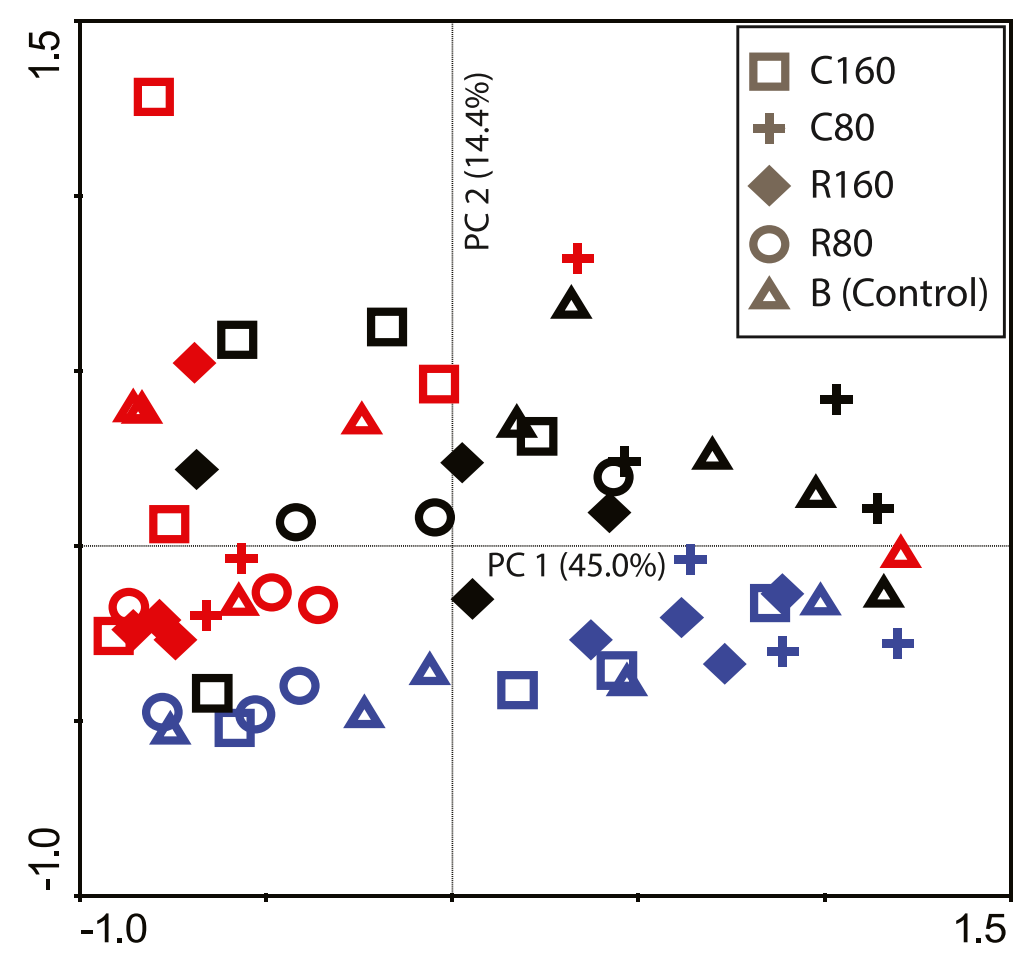

Fig. 1 Principal component analysis of Terminal restriction fragment length polymorphism data generated from the fecal microbiome. Symbols colored in blue, represents samples collected at weaning (D 0), in black, samples collected $17 \mathrm{~d}$ post weaning (D 17) and red, samples collected 35 days post weaning (D 35). Different symbols represent the different feed supplements, C; Chicory, R; Ribwort. Percentage values represent how much of the variation in data explained by each principal component (PC)

the segregation of samples were associated with the age of the pigs (Fig. 2). Comparing samples at D 0 and D 35 revealed a maturation of the gut microbiota with dramatic changes in the relative abundance of certain genera. Lactobacilli and enterobacteria were more abundant in younger pigs $(P<0.05)$, whereas the genera Streptococcus, Treponema, Clostridium, Clostridiaceae1 and Coprococcus were present in higher abundances in older pigs $(P<0.05$; Fig. 3$)$. In agreement with the T-RFLP data analysis, the $16 \mathrm{~S}$ data showed that individual pig samples collected at D 0 had a larger variation in the community structure than the samples collected at D 35 (Fig. 2).

\section{Diet dependent influences on the microbiome}

Despite the large temporal variation of the developing microbiota it was possible to identify changes that could be linked to inclusion of chicory or ribwort. As indicated earlier, the abundance of lactobacilli was reduced in samples collected at D 35. The reduction of lactobacilli was however significantly more pronounced in pigs fed the ribwort diet $(P=0.004$; Fig. 4$)$. Instead the pigs fed ribwort had a significantly increased abundance of sequences classified as Treponema ( $P=0.011$; Fig. 4). Another microbial group that was associated with the diet regime was Prevotellaceae. The abundance of Prevotellaceae decreased in animals fed the control diet whereas it increased in animals fed both the chicory and the ribwort diet $(P<0.001$; Fig. 4$)$.

\section{Correlations between digestion of dietary components and specific groups of microbes}

Pearson correlations were tested between abundance data from all microbial taxa from samples collected at D 35 and the digested amount of arabinose, xylose, mannose, galactose, glucose, uronic acid and total NSP from the same samples. We found a significant correlation between the abundance of Bacteroides and the digested amount of galactose and uronic acids (Table 4). In addition, the abundance of Bacteroides was also positively correlated with the digested amount of NSP, but the correlation did not reach significance (Table 4). Furthermore, the abundance of Bacteroidales was positively correlated with the digested amount of xylose (Table 4).

\section{Discussion}

The fecal microbiome changed dramatically in composition after weaning, regardless of diet, but inclusion of the different fiber sources had an impact on the development of the post weaning microbiome. Inclusion of ribwort had a larger effect on the post weaning microbiome compared to chicory (Fig. 4). The abundance of lactobacilli was lower in samples collected at weaning (D 0) compared with samples 


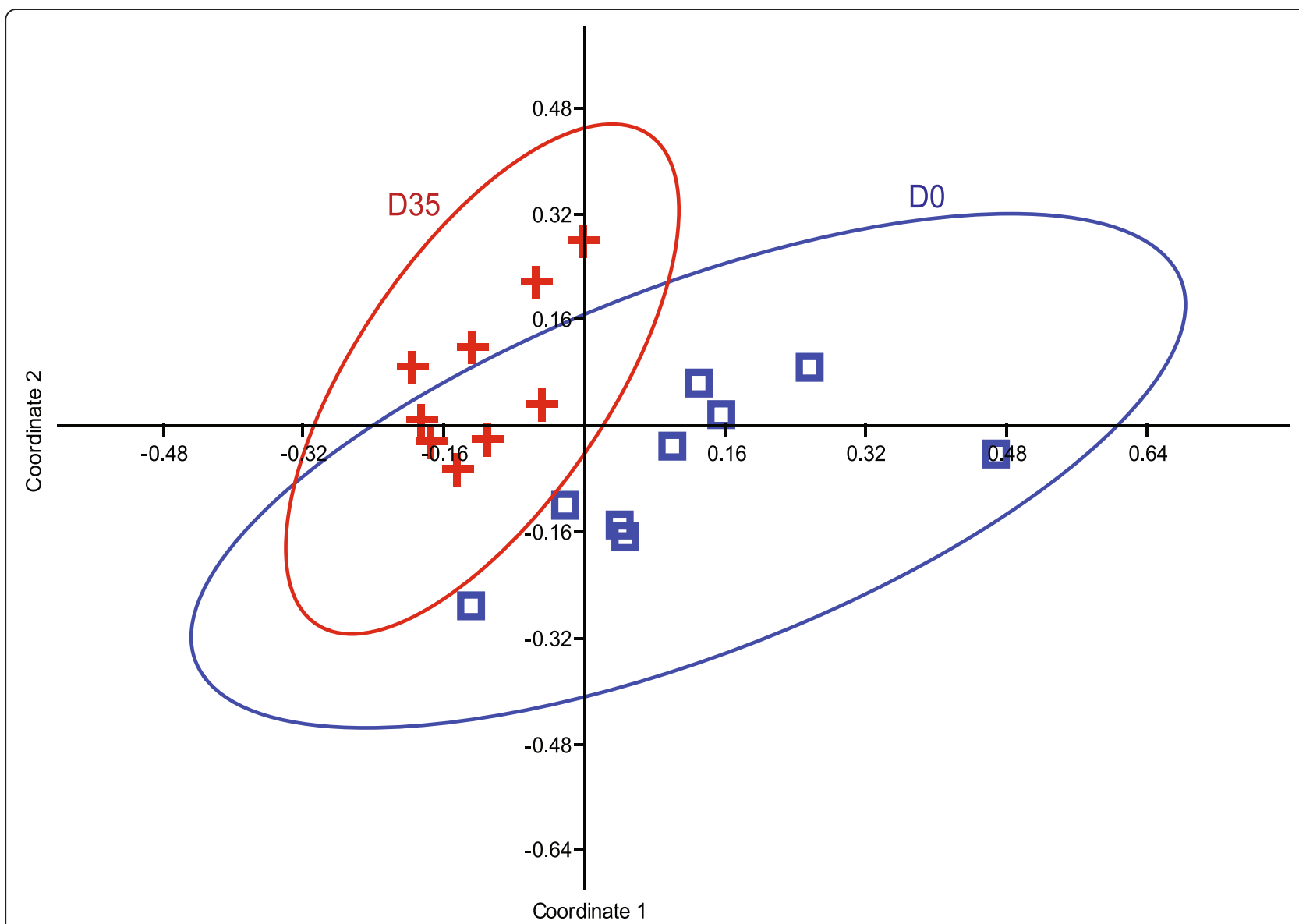

Fig. 2 Multivariate analysis of the $16 \mathrm{~S}$ sequence data. Principal coordinate analysis (PCoA) plot using Bray Curtis distances on the 165 sequence data classified down to genus level. Symbols colored in blue, represent samples collected at weaning (D 0) and red, samples collected 35 days post weaning (D 35)

collected 35 days post weaning (Fig. 3). However, for pigs fed the diet including ribwort, the abundance of lactobacilli had decreased to a larger extent compared with the other diets (Fig. 4). This indicates that ribwort inclusion has a negative impact on lactobacilli in the gut. Both chicory and ribwort have a high content of uronic acids, which derives from galactosyluronic acid and is a building block in pectins [6]. Pectin of plant origin, such as sugar beet pulp, has been used as a fibrous feedstuff in pig diets and resulted in an increased lactic acid bacteria (LAB) population in the small intestine [22]. In addition, we have earlier shown that inclusion of chicory forage was associated with higher abundances of LAB, primarily in ileal digesta, to a lesser extent in colonic digesta [11] but not in fecal samples [10]. In the present study, the chicory feed did not impact the fecal lactobacilli compared with the control. It is therefore likely that the effect of chicory forage on lactobacilli occurs primarily in the small bowel.

Our study showed that the abundance of Prevotella increased in pigs fed the chicory and ribwort diets compared to the control feed. Prevotella is one of the abundant bacteria found in the pig gut. This group of gram-negative bacteria is able to produce several xylanases, mannanases, $\beta$-glucanases, and corresponds to soluble xylan utilization, and is therefore likely important for biodegradation of complex sugars in the gut [23]. Rural African children and rural Papua New Guinea habitants, living on a fiber-rich diet harbor a gut microbiota rich in Prevotella spp. while this community is less abundant in European children and habitants in the United States, living on a 'Western' diet (typically high in animal protein, sugar, starch, and fat and low in fiber) [24, 25]. This indicates that the abundance of Prevotella is influenced by the fiber content in the diet but the type of fiber is also important. For example, it was shown that ruminal Prevotella ruminicola and Prevotella bryanti responded in opposite directions to hay and grain-based diets [26].

In the current dataset, the abundance of Bacteroides was positively correlated with the digested amount of galactose and uronic acid. In addition, the abundance of sequences classified as Bacteroidales was correlated with the digested amount of xylose. Bacteroides and Prevotella 


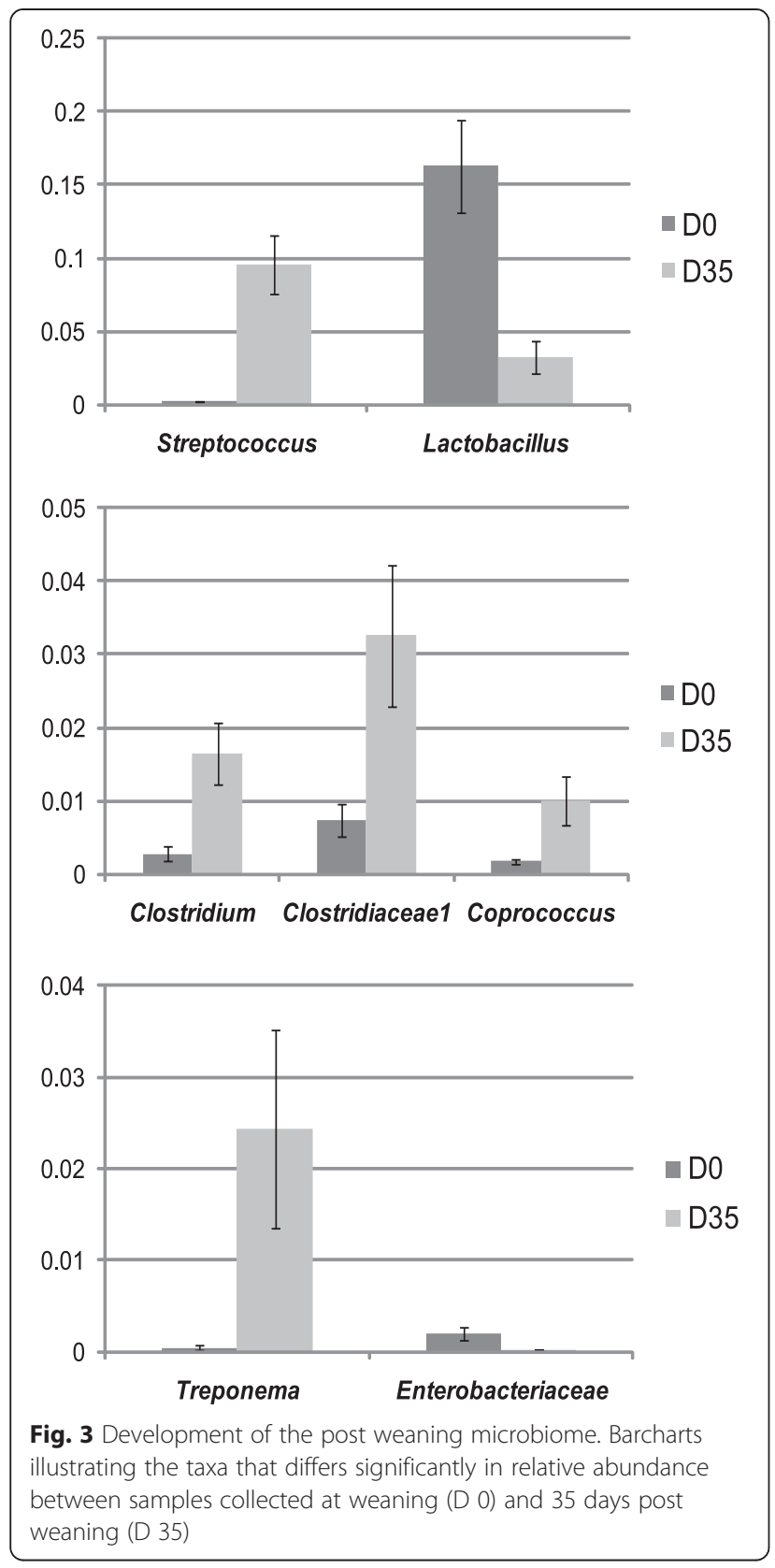

are the major carbohydrate degrading organisms in the gut and it is therefore not surprising that these positive correlations were found. Uronic acid is extensively fermented in the colon, but utilization of uronic acid is restricted to few genera. Bacteroides have the ability to utilize uronic acid $[27,28]$ as too do Faecalibacterium $[11,29]$. We could, however, not find a significant correlation between the abundance of Faecalibacterium and the digested amount of uronic acid.

The pigs fed the highest inclusion of ribwort had a significantly lower feed intake and weight gain compared with pigs fed the other diets. However, it is not known if the reduced weight gain and feed intake influenced the

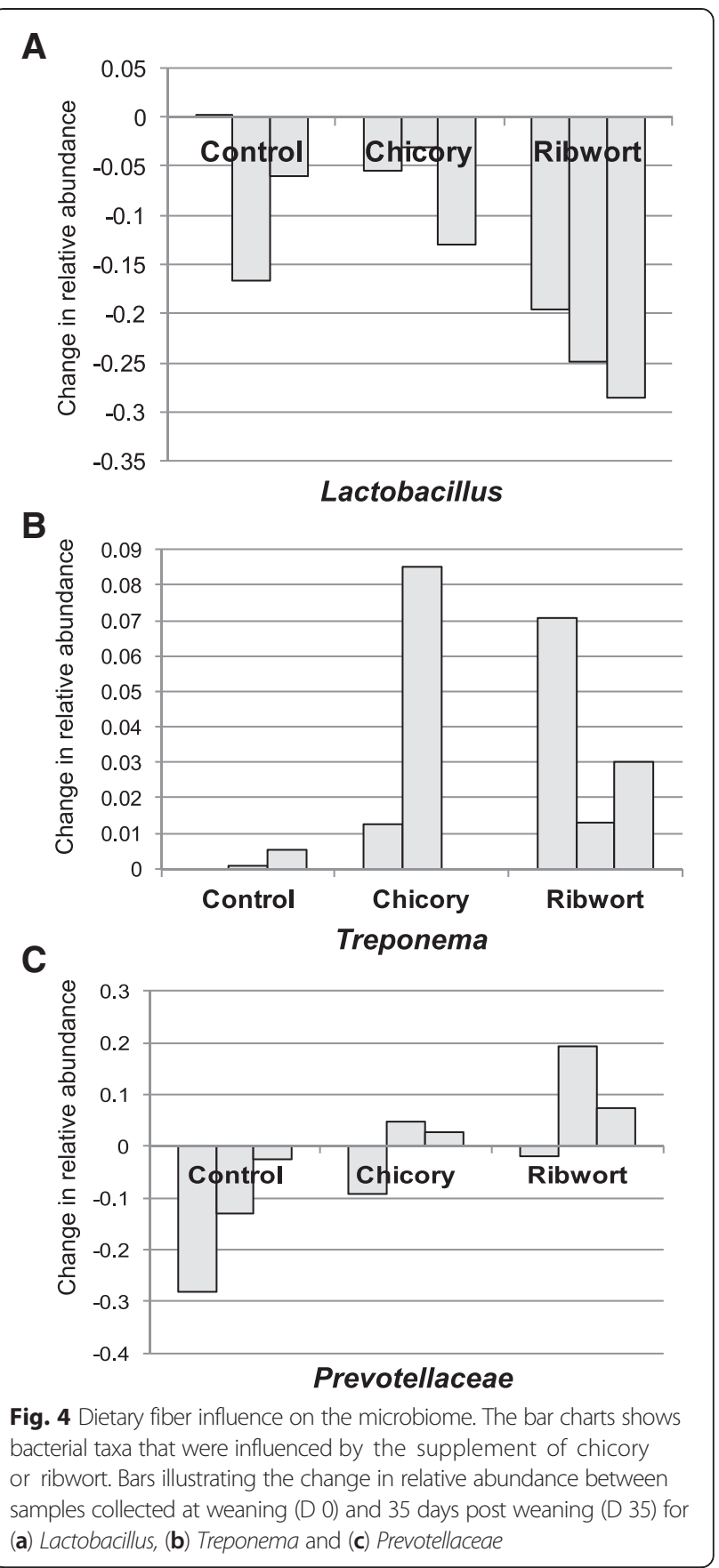

Table 4 Pearson correlations between bacterial abundance data and digested amount of dietary compounds

\begin{tabular}{llll}
\hline Variable & With variable & $r$ & $p$-value \\
\hline Bacteroides & galactose & 0.745 & 0.021 \\
Bacteroides & uronic acid & 0.688 & 0.041 \\
Bacteroides & NSP & 0.648 & 0.059 \\
Bacteroidales & xylose & 0.678 & 0.045 \\
\hline
\end{tabular}

Abbreviations: NSP non-starch polysaccharides 
microbiome. Neither is it possible to conclude to what extent the dietary influence in the microbiota structure was masked by the natural change in microbiota structure after weaning. The development of the post weaning microbiome was characterized by a dramatic shift in the bacterial composition with a marked reduction of lactobacilli and Enterobacteriaceae, and an increased abundance of the genera Streptococcus, Clostridium, Clostridiaceae1, Treponema, and Coprococcus. These bacterial groups are commonly detected in weaned pigs reflecting that the fecal microbiome in the pigs included in this study has a composition similar to what others have shown [30-32]. The dramatic change in the microbiome during weaning is in agreement with earlier studies in human infants [33, 34], and in previous studies in pigs that have shown a shift from a Lactobacillus dominated microbial population towards dominance of Streptococcus [35, 36]. In addition, the reduction in relative abundance of Enterobactericeae with increasing age was in agreement with earlier culturing data from the same animals [7].

\section{Conclusion}

In conclusion, this study demonstrated that both chicory and ribwort inclusion as feed supplements in the diet of newly weaned pigs, influenced the composition of the fecal microbiome. The feed supplements were associated with a change in the abundance of Lactobacillus, Treponema and Prevotella. Furthermore, we showed that digestion of specific dietary components was correlated with the species composition of the microbiota. However, the most dramatic change in the microbiota was found when fecal samples collected 17 and 35 days post weaning were compared with samples collected at weaning and demonstrated that the gut will be exposed to a dramatic shift in the microbial community structure several weeks after weaning.

\section{Competing interests}

The authors have no competing interests to declare.

\section{Authors' contributions}

JEL and JKJ conceived and designed the study. JD performed the laboratory analyses. JD and JEL analyzed the data. JD and JEL wrote the manuscript. JD, JKJ and JEL have read, provided feedback and approved the final version of the manuscript.

\section{Acknowledgements}

This study was funded by FORMAS, project no: 2005-1608. We thank Anna-Greta Haglund for skilled laboratory assistance and Zongli Zheng at the Department of Medical Epidemiology and Biostatistics, Karolinska Institute, Sweden, for advice and assistance in the statistical analysis.

\section{Author details}

'Department of Animal Nutrition and Management, Swedish University of Agricultural Sciences, P.O. Box 7024, SE75007 Uppsala, Sweden. ²Department of Microbiology, Swedish University of Agricultural Sciences, P.O. Box 7025, SE75007 Uppsala, Sweden. ${ }^{3}$ Division of Biology Earth and Biological Sciences, Pacific Northwest National Laboratories, P.O. Box 999, MSIN J4-18, WA99352 Richland, WA, USA.
Received: 2 July 2015 Accepted: 2 December 2015

Published online: 18 December 2015

\section{References}

1. Wenk C. The role of dietary fibre in the digestive physiology of the pig. Anim Feed Sci Tech. 2001;90:21-33.

2. Högberg A, Lindberg JE. The effect of level and type of cereal non-starch polysaccharides on the performance, nutrient utilization and gut environment of pigs around weaning. Anim Feed Sci Tech. 2006;127:200-19.

3. Högberg A, Lindberg JE. Influence of cereal non-starch polysaccharides and enzyme supplementation on digestion site and gut environment in weaned piglets. Anim Feed Sci Tech. 2004;116:113-28.

4. Gibson GR, Roberfroid MB. Dietary modulation of the human colonic microbiota: introducing the concept of prebiotics. J Nutr. 1995;125:1401-12.

5. Jorgensen $H$, Serena A, Hedemann MS, Knudsen KEB. The fermentative capacity of growing pigs and adult sows fed diets with contrasting type and level of dietary fibre. Livest Sci. 2007;109:111-4.

6. Voragen F, Beldman G, Schols H. Chemistry and enzymology of pectins. In: McCleary BV, Prosky L, editors. Advanced dietary fibre technology. Oxford, UK: Blackwell Science Ltd; 2001. p. 379-98.

7. Ivarsson E, Frankow-Lindberg BE, Andersson HK, Lindberg JE. Growth performance, digestibility and faecal coliform bacteria in weaned piglets fed a cereal-based diet including either chicory (Cichorium intybus L) or ribwort (Plantago lanceolata L) forage. Animal. 2011;5:558-64.

8. Leming R, Lindberg JE. Digestion of carbohydrates in fibre-rich diets for pigs. Agraarteadus. 2001;12:210-8.

9. Wang JF, Zhu YH, Li DF, Wang M, Jensen BB. Effect of type and level of dietary fibre and starch on ileal and faecal microbial activity and short-chain fatty acid concentrations in growing pigs. Anim Sci. 2004;78:109-17.

10. Ivarsson E, Liu HY, Dicksved J, Roos S, Lindberg JE. Impact of chicory inclusion in a cereal-based diet on digestibility, organ size and faecal microbiota in growing pigs. Animal. 2012;6:1077-85.

11. Liu H, Ivarsson E, Dicksved J, Lundh T, Lindberg JE. Inclusion of chicory (Cichorium intybus L.) in pigs' diets affects the intestinal microenvironment and the gut microbiota. Appl Environ Microbiol. 2012;78:4102-9.

12. Ivarsson E, Roos S, Liu HY, Lindberg JE. Fermentable non-starch polysaccharides increases the abundance of Bacteroides-PrevotellaPorphyromonas in ileal microbial community of growing pigs. Animal. 2014;8:1777-87.

13. Tamura $Y$, Nishibe $S$. Changes in the concentrations of bioactive compounds in plantain leaves. J Agr Food Chem. 2002;50:2514-8.

14. OIE (Office International des Epizooties). Old classification of diseases notifiable to the OIE. 2004. Retreived 15 April 2009, from http://www.oie.int/ eng/maladies/en_OldClassification.htm.

15. Leser TD, Amenuvor JZ, Jensen TK, Lindecrona RH, Boye M, Moller K. Culture-independent analysis of gut bacteria: the pig gastrointestinal tract microbiota revisited. Appl Environ Microbiol. 2002;68:673-90.

16. Edwards U, Rogall T, Blocker H, Emde M, Bottger EC. Isolation and direct complete nucleotide determination of entire genes-characterization of a gene coding for 16S-Ribosomal RNA. Nucl Acids Res. 1989;17:7843-53.

17. Muyzer G, Dewaal EC, Uitterlinden AG. Profiling of complex microbialpopulations by denaturing gradient gel-electrophoresis analysis of polymerase chain reaction-amplified genes-coding for 165 ribosomal-RNA. Appl Environ Microbiol. 1993;59:695-700.

18. Dicksved J, Flöistrup H, Bergström A, Rosenquist M, Pershagen G, Scheynius A, et al. Molecular fingerprinting of the fecal microbiota of children raised according to different lifestyles. Appl Environ Microbiol. 2007;73:2284-9.

19. Andersson AF, Lindberg M, Jakobsson H, Bäckhed F, Nyren P, Engstrand L. Comparative analysis of human gut microbiota by barcoded pyrosequencing. PLoS One. 2008;3:e2836.

20. Hammer O, Harper DAT, Ryan DT. Paleontological statistics software package for education and data analysis. Paleontologia Electronica. 2001:4:1-9.

21. Benjamini Y, Hochberg Y. Controling the false discovery rate: a practical and powerful approach to multiple testing. J R Stat Soc Ser B. 1995;57:289-300.

22. Konstantinov SR, Awati A, Smidt H, Williams BA, Akkermans ADL, De Vos WM. Specific response of a novel and abundant Lactobacillus amylovorus-like phylotype to dietary prebiotics in the guts of weaning piglets. Appl Environ Microbiol. 2004;70:3821-30.

23. Flint HJ, Bayer EA. Plant cell wall breakdown by anaerobic microorganisms from the mammalian digestive tract. Ann N Y Acad Sci. 2008;1125:280-8. 
24. De Filippo C, Cavalieri D, Di Paola M, Ramazzotti M, Poullet JB, Massart S, et al. Impact of diet in shaping gut microbiota revealed by a comparative study in children from Europe and rural Africa. Proc Natl Acad Sci. 2010;107:14691-6.

25. Martinez I, Stegen JC, Maldonado-Gomez MX, Eren AM, Siba PM, Greenhill AR, et al. The gut microbiota of rural Papua New Guineans: composition, diversity patterns, and ecological processes. Cell Rep. 2015;11:527-38.

26. Tajima K, Aminov Rl, Nagamine T, Matsui H, Nakamura M, Benno Y. Diet-dependent shifts in the bacterial population of the rumen revealed with real-time PCR. Appl Environ Microbiol. 2001;67:2766-74.

27. Salyers AA, Vercellotti JR, West SE, Wilkins TD. Fermentation of mucin and plant polysaccharides by strains of Bacteroides from the human colon. Appl Environ Microbiol. 1977;33(2):319-22.

28. Salyers AA, West SE, Vercellotti JR, Wilkins TD. Fermentation of mucins and plant polysaccharides by anaerobic bacteria from the human colon. Appl Environ Microbiol. 1977;34:529-33.

29. Lopez-Siles M, Khan TM, Duncan SH, Harmsen HJ, Garcia-Gil LJ, Flint HJ. Cultured representatives of two major phylogroups of human colonic Faecalibacterium prausnitzii can utilize pectin, uronic acids, and host-derived substrates for growth. Appl Environ Microbiol. 2012;78:420-8.

30. Kim HB, Borewicz K, White BA, Singer RS, Sreevatsan S, Tu ZJ, et al. Longitudinal investigation of the age-related bacterial diversity in the feces of commercial pigs. Vet Microbiol. 2011;153:124-33.

31. Niu Q, Li P, Hao S, Zhang Y, Kim SW, Li H, et al. Dynamic distribution of the gut microbiota and the relationship with apparent crude fiber digestibility and growth stages in pigs. Sci Rep. 2015;5:9938.

32. Zhao W, Wang Y, Liu S, Huang J, Zhai Z, He C, et al. The dynamic distribution of porcine microbiota across different ages and gastrointestinal tract segments. PLoS One. 2015;10, e0117441.

33. Bäckhed F, Roswall J, Peng Y, Feng $Q$, Jia H, Kovatcheva-Datchary $P$, et al dynamics and stabilization of the human gut microbiome during the first year of life. Cell Host Microbe. 2015;17:852.

34. Hesla HM, Stenius F, Jäderlund L, Nelson R, Engstrand L, Alm J, et al. Impact of lifestyle on the gut microbiota of healthy infants and their mothers-the ALADDIN birth cohort. FEMS Microbiol Ecol. 2014;90:791-801.

35. Konstantinov SR, Awati AA, Williams BA, Miller BG, Jones $P$, Stokes $C R$, et al. Post-natal development of the porcine microbiota composition and activities. Environ Microbiol. 2006:8:1191-9.

36. Su Y, Yao W, Perez-Gutierrez ON, Smidt H, Zhu WY. Changes in abundance of Lactobacillus spp. and Streptococcus suis in the stomach, jejunum and ileum of piglets after weaning. FEMS Microbiol Ecol. 2008;66:546-55.

\section{Submit your next manuscript to BioMed Central and we will help you at every step:}

- We accept pre-submission inquiries

- Our selector tool helps you to find the most relevant journal

- We provide round the clock customer support

- Convenient online submission

- Thorough peer review

- Inclusion in PubMed and all major indexing services

- Maximum visibility for your research 